\title{
Incidence of Post-Procedural COVID-19 at an Urban Academic Medical Center during High Prevalence Months
}

\author{
Joanne Szewczyk, MDㄹ, Dao M Nguyen, MD, MSc', Prem R Warde, MSIE ${ }^{2}$, Bhavarth \\ Shukla, MD, $\mathrm{MPH}^{3}$, Tanira Ferreira, $\mathrm{MD}^{4 *}$, Dipen J Parekh, $M D^{5,6}$ and Hayley $B$ \\ Gershengorn, $M D^{4}$
}

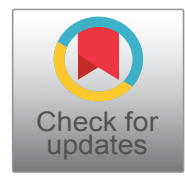

${ }^{1}$ Thoracic Surgery Section, Division of Cardiothoracic Surgery, The DeWitt Daughtry Department of Surgery, University of Miami Miller School of Medicine, USA

${ }^{2}$ Care Transformation, University of Miami Hospital and Clinics, USA

${ }^{3}$ Division of Infectious Diseases, Department of Medicine, University of Miami Miller School of Medicine, USA

${ }^{4}$ Division of Pulmonary, Critical Care, and Sleep Medicine, Department of Medicine, University of Miami Miller School of Medicine, USA

${ }^{5}$ Department of Urology, University of Miami Miller School of Medicine, USA

${ }^{6}$ University of Miami Health System, USA

*Corresponding author: Tanira Ferreira, MD, Division of Pulmonary, Critical Care, and Sleep Medicine, Department of Medicine, University of Miami Miller School of Medicine, 1400 NW $12^{\text {th }}$ Avenue, Suite 2022, Miami, Florida 33136, USA

\begin{abstract}
In the beginning of the pandemic, after elective procedures resumed in Florida, we tested all patients for COVID-19, nasal pharyngeal swab PCR 72 hrs prior to any procedure. We conducted a retrospective cohort to see the incidence of COVID-19 post-procedural.

Methods: We conducted a retrospective cohort study of all adult cases of same day or hospital-based procedures at the University of Miami Hospital and Clinics from April 1-September 23, 2020 who were COVID-19-negative (by SARS-CoV-2 polymerase chain reaction testing) within 72 hours pre-procedure, had contact with our health system 5-14 days post-procedure, and were either screened for COVID-19 symptoms or tested for SARS-CoV-2 during this contact. This timeframe was selected as median time to symptom onset after exposure is 5 days and $99 \%$ are symptomatic by 14 days.
\end{abstract}

Results: The cohort consisted of 8,881 cases of which $879(9.9 \%)$ were both screened for symptoms and tested for SARS-CoV-2 within 5-14 days post-procedure; 5,748 $(64.7 \%)$ were screened but not tested and $131(1.5 \%)$ were tested but not screened (Figure 1 and Table 1).

Overall, 82 post-procedure screens (1.2\% of screens) revealed COVID-19-associated symptoms and 13 tests $(1.3 \%$ of tests) were positive; 91 (1.0\% of all cases) had either symptoms or a test consistent with COVID-19. A positive symptom screen was only $40.0 \%$ sensitive, but $94.9 \%$ specific for a positive COVID-19 test; negative predictive value was $99.3 \%$.

Conclusion: COVID-19 acquisition post procedure demonstrates a strikingly low rate of infection $(<1.5 \%)$. As Miami was one of the pandemic epicenters during the period of our study, this finding provides reassurance that proceeding with hospital-based procedures is quite safe even with high community COVID-19 prevalence. This is especially important right now as COVID-19 surges across the nation.

\section{Introduction}

The coronavirus disease of 2019 (COVID-19) has created unprecedented challenges to health care. In the spring, many elective procedures were postponed to decrease potential exposures and allow resource re-allocation [1]. As elective procedures resume, we face a new challenge-patient fear. Two-fifths of U.S. adults report avoiding or delaying care out of concern for COVID-19 [2]. While current literature suggests hospital acquired COVID-19 is rare, little is known about the

Citation: Szewczyk J, Nguyen DM, Warde PR, Shukla B, Ferreira T, et al. (2021) Incidence of PostProcedural COVID-19 at an Urban Academic Medical Center during High Prevalence Months. Int J Respir Pulm Med 8:150. doi.org/10.23937/2378-3516/1410150

Accepted: March 29, 2021; Published: March 31, 2021

Copyright: (C) 2021 Szewczyk J, et al. This is an open-access article distributed under the terms of the Creative Commons Attribution License, which permits unrestricted use, distribution, and reproduction in any medium, provided the original author and source are credited 


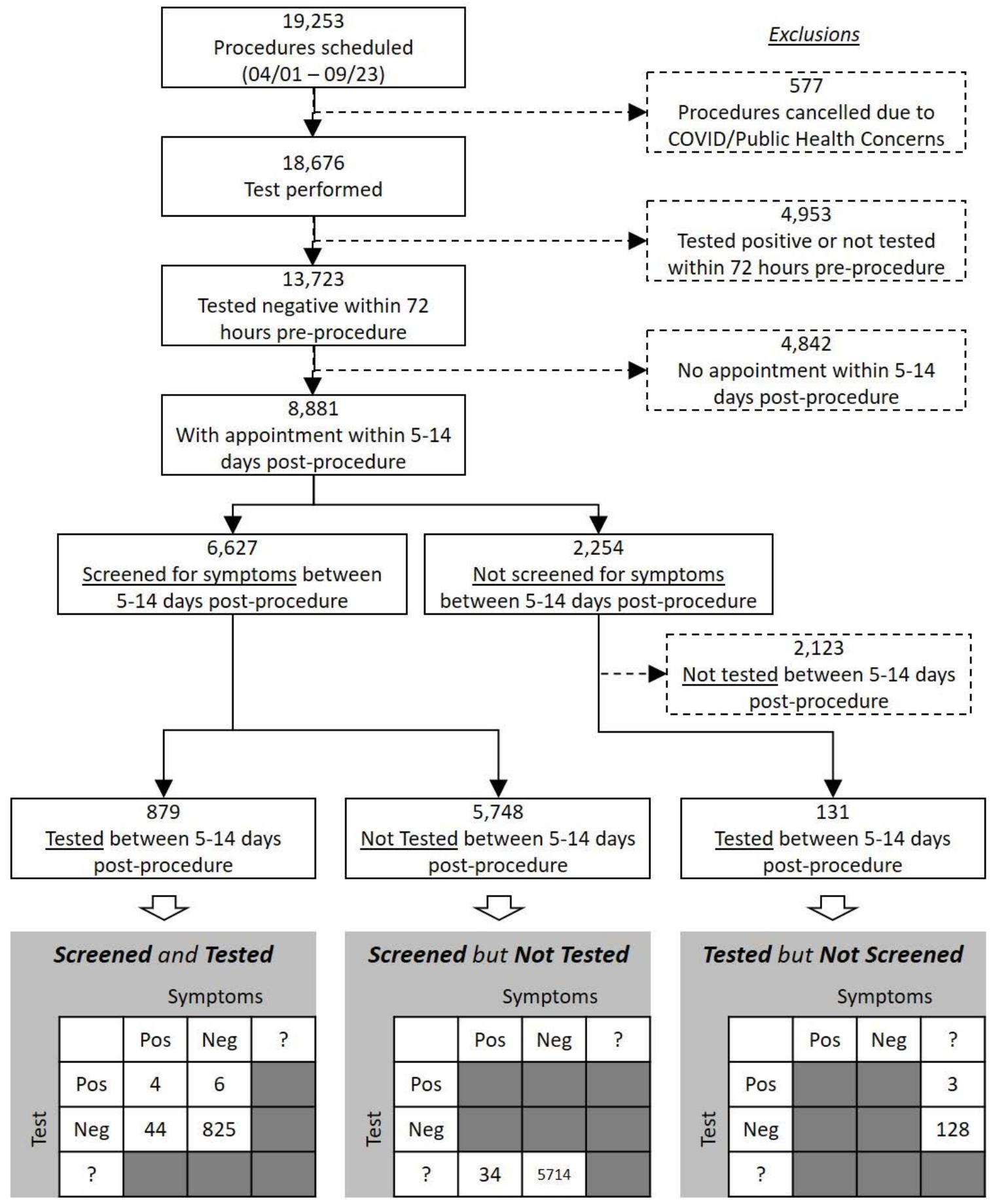

Figure 1: Cohort flow diagram with symptom screen and testing results.

Neg: Negative; Pos: Positive; ?: Unknown

risk to patients undergoing same day or hospital-based procedures [3]. In this study we investigated the rate of post-procedural COVID-19 and hypothesized it would be low.

\section{Methods}

We conducted a retrospective cohort study of all adult cases of same day or hospital-based procedures at the University of Miami Hospital and Clinics from April 1-September 23, 2020 who were COVID-19-negative (by SARS-CoV-2 polymerase chain reaction testing) within
72 hours pre-procedure, had contact with our health system 5-14 days post-procedure, and were either screened for COVID-19 symptoms or tested for SARSCoV-2 during this contact. This time frame was selected as median time to symptom onset after exposure is 5 days and $99 \%$ are symptomatic by 14 days [4].

For all cases, case-specific data, results of all tests, and answers to symptom screens (intended to be performed at each health system contact) were obtained. Standard statistics were used to describe cohort characteristics and post-procedural symptom screen and test- 
ing outcomes. Among cases who were both screened and tested, we evaluated the accuracy of symptom screening for test positivity. Finally, for cases who had symptoms on screening but were never tested within our system, we performed a qualitative chart review to understand the circumstances. This study was approved by the Institutional Review Board from the University of Miami (\#20200739).

\section{Results}

The cohort consisted of 8,881 cases of which 879 (9.9\%) were both screened for symptoms and tested for SARS-CoV-2 within 5-14 days post-procedure; 5,748 $(64.7 \%)$ were screened but not tested and 131 (1.5\%) were tested but not screened (Figure 1 and Table 1).

Overall, 82 post-procedure screens (1.2\% of screens)

Table 1: Cohort characteristics.

\begin{tabular}{|c|c|c|c|c|}
\hline Characteristic & $\begin{array}{l}\text { Screened and } \\
\text { Tested, } \\
\text { N (\%) }\end{array}$ & $\begin{array}{l}\text { Screened but not } \\
\text { Tested, } \\
\text { N (\%) }\end{array}$ & $\begin{array}{l}\text { Tested but not } \\
\text { Screened, } \\
\text { N (\%) }\end{array}$ & $\begin{array}{l}\text { Not Screened or } \\
\text { Tested, } \\
\text { N (\%) }\end{array}$ \\
\hline \# Of cases, $\mathrm{N}$ (row \%) & $879(9.9 \%)$ & $5.748(65 \%)$ & $131(1.5 \%)$ & $2.123(24 \%)$ \\
\hline $\begin{array}{l}\text { Days from pre-procedure negative } \\
\text { test to procedure, median (IQR) }\end{array}$ & $1.83(1.00,2.04)$ & $1.92(1.00,2.08)$ & $1.12(0.71,1.94)$ & $1.92(1.00,2.12)$ \\
\hline $\begin{array}{l}\text { Days from procedure to post- } \\
\text { procedure symptoms screen, } \\
\text { median (IQR) }\end{array}$ & $7.79(5.98,10.75)$ & $8(6.83,10.92)$ & $\mathrm{n} / \mathrm{a}$ & $\mathrm{n} / \mathrm{a}$ \\
\hline $\begin{array}{l}\text { Days from procedure to post- } \\
\text { procedure test, median (IQR) }\end{array}$ & $9.88(6.08,11.96)$ & $\mathrm{n} / \mathrm{a}$ & $7.88(6.75,10.08)$ & $\mathrm{n} / \mathrm{a}$ \\
\hline Age (years), median (IQR) & $65(55,73)$ & $60(49,70)$ & $68(60,76)$ & $60(48,70)$ \\
\hline \multicolumn{5}{|l|}{ Race/ethnicity } \\
\hline Hispanic-black & $12(1.4 \%)$ & $86(1.5 \%)$ & $2(1.5 \%)$ & $35(1.6 \%)$ \\
\hline Hispanic-white & $349(40 \%)$ & $2.402(42 \%)$ & $50(38 \%)$ & $885(42 \%)$ \\
\hline Non-hispanic-black & $107(12 \%)$ & $726(13 \%)$ & $24(18 \%)$ & $275(13 \%)$ \\
\hline Non-hispanic-white & $295(34 \%)$ & $1.651(29 \%)$ & $40(31 \%)$ & $622(29 \%)$ \\
\hline Other & $32(3.6 \%)$ & $238(4.1 \%)$ & $7(5.3 \%)$ & $83(3.9 \%)$ \\
\hline Unknown & $84(9.6 \%)$ & $645(11 \%)$ & $8(6.1 \%)$ & $223(11 \%)$ \\
\hline Female gender & $461(52 \%)$ & $2.785(48 \%)$ & $63(48 \%)$ & $1.120(53 \%)$ \\
\hline \multicolumn{5}{|l|}{ Payer } \\
\hline Commercial & $357(41 \%)$ & $2.780(48 \%)$ & $42(32 \%)$ & $1.059(50 \%)$ \\
\hline Government & $29(3.3 \%)$ & $268(4.7 \%)$ & $1(0.8 \%)$ & $36(1.7 \%)$ \\
\hline Medicaid & $63(7.2 \%)$ & $429(7.5 \%)$ & $8(6.1 \%)$ & $191(9.0 \%)$ \\
\hline Medicare & $418(48 \%)$ & $2.110(37 \%)$ & $76(58 \%)$ & $807(38 \%)$ \\
\hline Other & $12(1.4 \%)$ & $161(2.8 \%)$ & $4(3.1 \%)$ & $30(1.4 \%)$ \\
\hline \multicolumn{5}{|l|}{ Service line } \\
\hline Cardiology & $34(3.9 \%)$ & $96(1.7 \%)$ & $8(6.1 \%)$ & $99(4.7 \%)$ \\
\hline Otolaryngologic surgery & $36(4.1 \%)$ & $628(11 \%)$ & $10(7.6 \%)$ & $150(7.1 \%)$ \\
\hline Gastroenterology & $149(17 \%)$ & $663(12 \%)$ & $35(27 \%)$ & $459(22 \%)$ \\
\hline General & $13(1.5 \%)$ & $98(1.7 \%)$ & $7(5.3 \%)$ & $108(5.1 \%)$ \\
\hline Neurosurgery & $29(3.3 \%)$ & $251(4.4 \%)$ & $13(9.9 \%)$ & $125(5.9 \%)$ \\
\hline Ophthalmology & $459(52 \%)$ & $2.642(46 \%)$ & $10(7.6 \%)$ & $518(24 \%)$ \\
\hline Orthopaedic surgery & $14(1.6 \%)$ & $239(4.2 \%)$ & $5(3.8 \%)$ & $84(4.0 \%)$ \\
\hline Surgical oncology & $31(3.5 \%)$ & $194(3.4 \%)$ & $2(1.5 \%)$ & $97(4.6 \%)$ \\
\hline Urology & $33(3.8 \%)$ & $281(4.9 \%)$ & $7(5.3 \%)$ & $145(6.8 \%)$ \\
\hline Other ${ }^{a}$ & $81(9.2 \%)$ & $656(11 \%)$ & $34(26 \%)$ & $338(16 \%)$ \\
\hline
\end{tabular}

aServices lines which contributed $<2.5 \%$ of total cases are combined, including: Anesthesiology, Cardiothoracic urgery, Colorectal

Surgery, Dermatology, Endocrinology, Gynaecology, Gynaecology-Oncology, Gynaecology-Urology, Hepatology, Oculoplastic Surgery, Oral Surgery, Pain, Plastic Surgery, Podiatry, Pulmonary, Radiation Oncology, Sports Medicine, Thoracic Surgery, and Vascular Surgery.

Abbreviations: Interquartile Range (IQR); not applicable (n/a). 
revealed COVID-19-associated symptoms and 13 tests (1.3\% of tests) were positive; 91 (1.0\% of all cases) had either symptoms or a test consistent with COVID-19. A positive symptom screen was only $40.0 \%$ sensitive, but 94.9\% specific for a positive COVID-19 test; negative predictive value was $99.3 \%$.

Chart review of the 34 cases ( $0.4 \%$ of all cases) who screened positive for symptoms but were not tested revealed flaws in the screening process. Cases falsely screened positive by acknowledging prior COVID-19 testing (often pre-operative, not symptom-triggered) or, less frequently, reporting symptoms which were chronic (not due to COVID-19).

\section{Discussion}

We found a rate of post-procedure COVID-19 acquisition of $<1.5 \%$, even lower than daily test positivity rates for Florida during the same time period of $2.3 \%$ $19.6 \%$ [5]. Our health system has strict infection control practices-testing all patients prior to hospital admission, separate COVID-19 inpatient wards, individual rooms with droplet precautions for all patients, adequate personal protective equipment-which likely minimized nosocomial COVID-19 transmission. As community exposure is possible pre-procedurally (between testing and the procedure) or post-procedurally (before follow-up at day 5-14), we provide COVID-19 prevention education on discharge.

Our study was limited by its single center design which may impact generalizability. We also lacked access to test results performed outside our system. Lastly, $23.9 \%$ of cases were neither screened nor tested which may have introduced bias despite demographic and clinical similarities to included cases.

Our results suggest the risk of acquiring post-procedural COVID-19 is low in the setting of strict infection control practices. Further delay of procedures due to fear of contracting COVID-19 may not be warranted.

\section{Conflict of Interest}

The authors declare no conflicts of interest.

\section{Funding}

None of the authors received funding.

\section{References}

1. Søreide K, Hallet J, Matthews JB, Schnitzbauer AA, Line PD, et al. (2020) Immediate and long-term impact of the COVID-19 pandemic on delivery of surgical services. $\mathrm{Br} \mathrm{J}$ Surg 107: 1250-1261.

2. Czeisler MÉ, Marynak K, Clarke KEN, Salah Z, Shakya I, et al. (2020) Delay or avoidance of medical care because of COVID-19-related concerns-United States, June 2020. Morbidity and Mortality Weekly Report 69: 1250-1257.

3. Rhee C, Baker M, Vaidya V, Tucker R, Resnick A, et al. (2020) Incidence of nosocomial COVID-19 in patients hospitalized at a large us academic medical center. JAMA Netw Open 3.

4. Daily state-by-state testing trends. Johns Hopkins Coronavirus Resource Center.

5. Stephen AL, Kyra HG, Bi Q, Forrest KJ, Zheng Q, et al. (2020) The incubation period of coronavirus disease 2019 (COVID-19) from publicly reported confirmed cases: Estimation and application. Ann Intern Med 172: 577-582. 